\title{
Roboter im Bergbau - wo liegt der Bedarf?
}

\author{
Nikolaus A. Sifferlinger
}

Lehrstuhl für Bergbaukunde, Bergtechnik und Bergwirtschaft, Montanuniversität Leoben, Leoben, Österreich

Eingegangen 7. Dezember 2020; angenommen 11. Januar 2021; online publiziert 27. Januar 2021

\begin{abstract}
Zusammenfassung: Durch die rauen Umgebungsbedingungen untertage und die Gefahr von Steinfall oder sogar Gebirgsschlägen ist der Mensch im Bergbau immer einem Restrisiko ausgesetzt.

Um wirklich automatische Bereiche im Bergbau ohne Personenzutritt betreiben zu können, wird es notwendig sein, Roboter für die Installation, Wartung und Betrieb von Anlagen verfügbar zu haben, die den Menschen vor Ort ersetzen können.

Der Beitrag gibt eine kurze Übersicht über den Bedarf an Robotern im Bergbau, den derzeitigen Zustand und die Auswirkungen auf die Lehre und Forschung an der Montanuniversität Leoben.
\end{abstract}

Schlüsselwörter: Bergbau, Roboter, Sicherheit, Wartung

Robots in Mining-What Do We Need?

Abstract: The harsh environment conditions underground and the possible danger by roof fall or even rock bursts leave a residual risk to humans in mining.

In order to enable automated, unmanned operated areas in mining, robots for installation, maintenance, and operation will be necessary to replace human workforce on site.

This article gives a short overview of the robotic needs for mining, of the state-of-the-art, and the impact into education and research at Montanuniversitaet Leoben.

Keywords: Mining, Robots, Safety, Maintenance

\section{Einleitung}

Per Definition ist ein Roboter eine Maschine, die dem Menschen dient, um häufig wiederkehrende mechanische Ar-

\footnotetext{
Univ.-Prof. DI. Dr. mont. N. A. Sifferlinger $(\varangle)$

Lehrstuhl für Bergbaukunde, Bergtechnik und Bergwirtschaft,

Montanuniversität Leoben,

Erzherzog-Johann-Str. 3/5

8700 Leoben, Österreich

Nikolaus-august.sifferlinger@unileoben.ac.at
}

beiten zu übernehmen. Zu diesen Arbeiten gehören Aufgaben, die hochgenau ausgeführt werden müssen, aber auch Arbeiten in gefährlicher Umgebung. Dabei werden die Roboter von Rechnerprogrammen gesteuert, bei Bedarf kann auch die Fernsteuerung durch Menschen erfolgen.

Durch die rauen Umgebungsbedingungen untertage und die Gefahr von Steinfall oder sogar Gebirgsschlägen ist der Mensch im Berg- und Tunnelbau immer einem Restrisiko ausgesetzt. Dies ist der Haupttreiber für die Entwicklungen in Richtung vollständiger Mechanisierung und dann Automation des Bergbaubetriebs.

Um wirklich automatische Bereiche im Bergbau ohne Personenzutritt betreiben zu können, wird es notwendig sein, Roboter für die Installation, Wartung und Betrieb von Anlagen verfügbar zu haben, die den Menschen vor Ort ersetzen können.

Für den Einsatz im Untertage-Bergbau werden meist autonome mobile Roboter erforderlich sein.

Und ja - es gibt sie schon in Einzelfällen, Roboter im Bergbau, wie in Abschn. 4 gezeigt wird.

\section{Wo liegt der Bedarf für Roboter untertage?}

Bedingt durch den fortlaufenden Abbau der oberflächennahen Lagerstätten ist der Bergbau gezwungen, den Wertmineralvorkommen in tiefere Bereiche zu folgen.

Mit größerer Teufe nehmen auch die Herausforderungen durch Gebirgsdruck und Temperatur stark zu, entsprechend sind die Aufwendungen in Ausbau, Bewetterung, Transport und Kühlung. Bei wasserführenden Schichten ist auch die Wasserhaltung eine große Aufgabe.

Entsprechend schwieriger werden auch die Arbeitsbedingungen für das Personal untertage, und die Gewährleistung der Sicherheit wird auch immer aufwendiger.

Daher ist der Wunsch, den Menschen wo möglich durch Arbeitsmaschinen - Roboter - zu ersetzen, schon ein alter [1].

Die Treiber für Automation im Bergbau sind [2]:

- Die fordernde, raue Arbeitsumgebung 
- Mangel an qualifiziertem Personal

- Gesundheit und Sicherheit

- Reduktion der Wartung durch Überlastung

- Steigerung der Effizienz im Betrieb

- Nachhaltigkeit der Ressourcen-Nutzung

Die Hauptaufgaben für Roboter im Untertagebergbau lassen sich daher wie folgt aufzählen:

- Exploration

- Vortrieb

- Gewinnung

- Gebirgssicherung

- Einbau der Infrastruktur

- Trennung von Wert- und Taubmineralien möglichst vor Ort

- Transport

- Überwachung in gefährlichen Bereichen

- Search and Rescue - wenn Personen von Unfällen betroffen sind

- Wartungs- und Reparaturarbeiten

Wie in Abschn. 4 gezeigt wird, gibt es bereits einige Maschinen und Systeme im Bergbau, die automatisch oder ferngesteuert ihre Aufgabe versehen. So bietet das Sandvik AutoMine ${ }^{\circledR}$ System den autonomen und teleferngesteuerten Betrieb für seine Untertage-Trucks und LHDs (LoadHaulDump) an [3]. Die autonomen Fahrzeuge können untertage viele Kilometer völlig alleine zurücklegen und ihre Fracht abliefern. Für die Wartung und Reparatur dieser Maschinen ist aber immer noch der Mensch unersetzlich.

Daraus lässt sich ableiten. dass für den weitgehend mannlosen Betrieb im Untertagebergbau Roboter für die Montage, die Wartung und den Betrieb der Anlagen erforderlich sein werden.

\section{Roboter für die Montage, Wartung und Betrieb}

Während in Industriebetrieben, die eine klare und definierte bauliche Geometrie und entsprechend "saubere" Umgebung aufweisen, Roboter durchaus schon andere Roboter warten können, ist dies im Bergbau mit seinen schwierigen Umgebungsbedingungen noch lange nicht der Fall.

Für die Montage und Wartung im Bergbau werden hochmobile Roboter benötigt, die wie der Mensch durch das Bergbaugebäude und an und auf den Anlagen arbeiten können. Erst wenn Installation und Wartung durch Roboter erledigt werden können, wird ein mannloser Betrieb möglich werden.

Die Bergbauindustrie ist in Bezug auf das Forschungsund Entwicklungsbudget und Ingenieurskapazitäten gegenüber der Fahrzeug- oder Flugzeugindustrie um Größenordnungen kleiner und die Entwicklungen dauern daher auch länger

Trotzdem sind mit den autonomen Fahrzeugen für den Materialtransport im Bergbau beachtliche Entwicklungen gelungen.
Die Entwicklung mobiler Wartungsroboter überschreitet derzeit die Möglichkeiten der Bergbauindustrie - aber hier ergibt sich die Möglichkeit von den Entwicklungen der Militärindustrie zu profitieren, die ähnliche Anforderungen an ihre mobilen Roboter hat. In den USA, Russland und China werden viele Milliarden Euro in die Entwicklung mobiler Roboter für den Kampfeinsatz, aber auch für die Wartung investiert.

Humanoide Roboter eignen sich besonders für die Inspektion und Wartung sowie für Einsätze bei Unfällen, da sie schlechte Wegbedingungen und Hindernisse meistern können [4].

Rad- oder Kettenfahrwerk gebundene Roboter benötigen gute Bodenbedingungen zur Fortbewegung, daher sind für den Bergbau in schwierigen Situationen Beine besser zum Weiterkommen. Zwei Beine ermöglichen rasches Bewegen, vier Beine geben mehr Stabilität, brauchen aber auch mehr Platz.

Die Defence Advanced Research Projects Agency (DARPA) ist die Forschungs- und Entwicklungsagentur des US Verteidigungsministeriums. Sie ist verantwortlich für die Entwicklung neuer Technologien für die Verwendung im Militär. Bei 220 Mitarbeitern hatte sie 2019 ein Forschungsbudget von 3,427 Mrd. Dollar zu vergeben. Und ein großer Budgetanteil geht auch in die Entwicklung von Robotsystemen [5].

Im November 2020 fand die 3. DARPA Subterranean Challange statt, deren Aufgabe es für die 17 teilnehmenden Teams ist, innovative Lösungen für die Kartierung, Navigation und Untersuchung von simulierten Untertageumgebungen zu finden [6, 7]. Das US-Militär sieht einen Teil der zukünftigen Einsätze in Großstädten mit viel unterirdischer Infrastruktur und bereitet sich entsprechend vor. Und Roboter sollen dabei einen wesentlichen Teil der Aufgaben übernehmen [8].

Die US-Firma Boston Dynamics hat den humanoiden Roboter ATLAS und den vierbeinigen Roboter SPOT ${ }^{\circledR}$ entwickelt [9].

ATLAS hat eine Größe von 1,5m und ein Gewicht von $80 \mathrm{~kg}$ und kann schon autonom in der Landschaft oder in Gebäuden gehen (siehe Videos unter https://www. bostondynamics.com/atlas, diese sind sehenswert).

Ausgehend von den Entwicklungsfortschritten im vergangenen Jahrzehnt ist davon auszugehen, dass ein Nachfolger von ATLAS oder ähnlichen Prototypen in den nächsten zwanzig Jahren auch Arbeitsaufgaben im Bergbau übernehmen können wird.

\section{Roboter im Bergbau 2021}

Bereits heute sind eine ganze Reihe von Robotern im Bergbau im Einsatz, die Teilaufgaben erfüllen. In diesem Abschnitt werden einige beispielhaft angeführt. 
Abb. 1: Der im europäischen Forschungsprojekt HORIZON 2020 UNEXMIN entwickelte Explorationsroboter UX 1 im Sommer 2019 in der überfluteten Höhle Molnar Janos (Quelle: UNEXMIN https://www.unexmin.eu/imagegallery/)

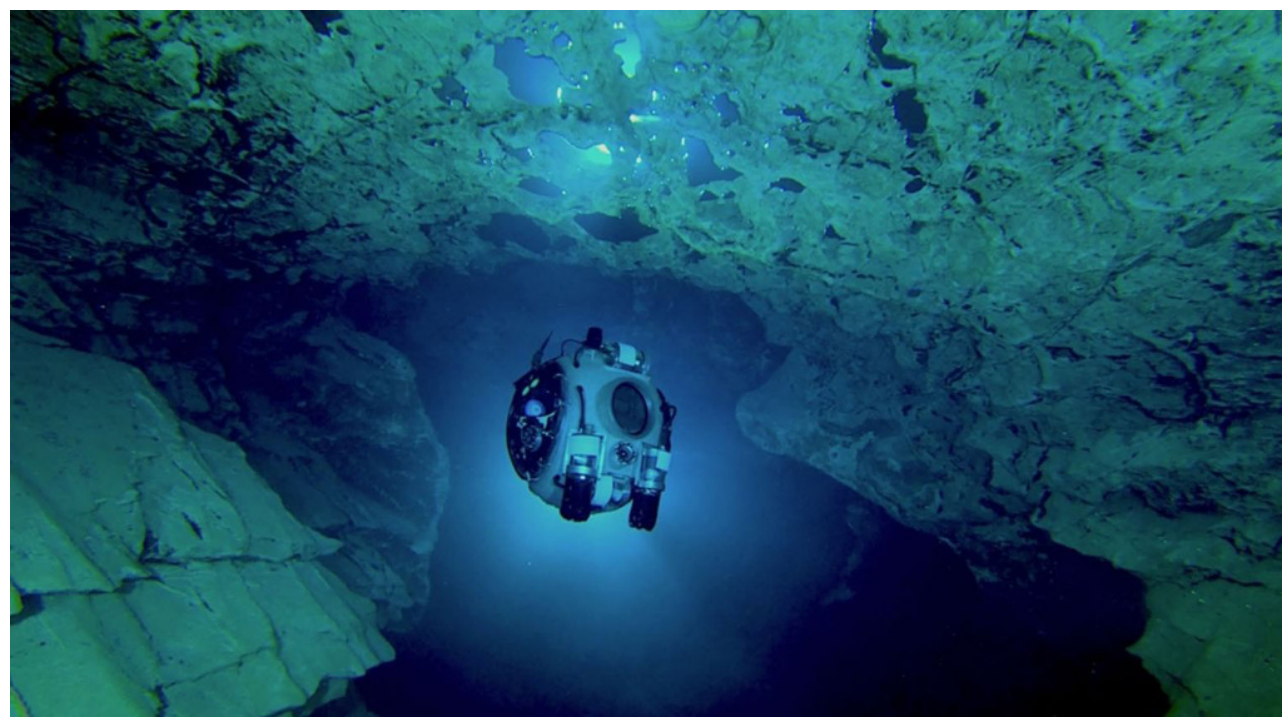

Abb. 2: Roboter SPOT ${ }^{\circledR}$ der US-Firma Boston Dynamics wird beim schwedischen Bergbaukonzern LKAB zur Erkundung in gefährlichen Zonen erprobt (Quelle: Boston Dynamics https://www.bostondynamics.com/ spot)

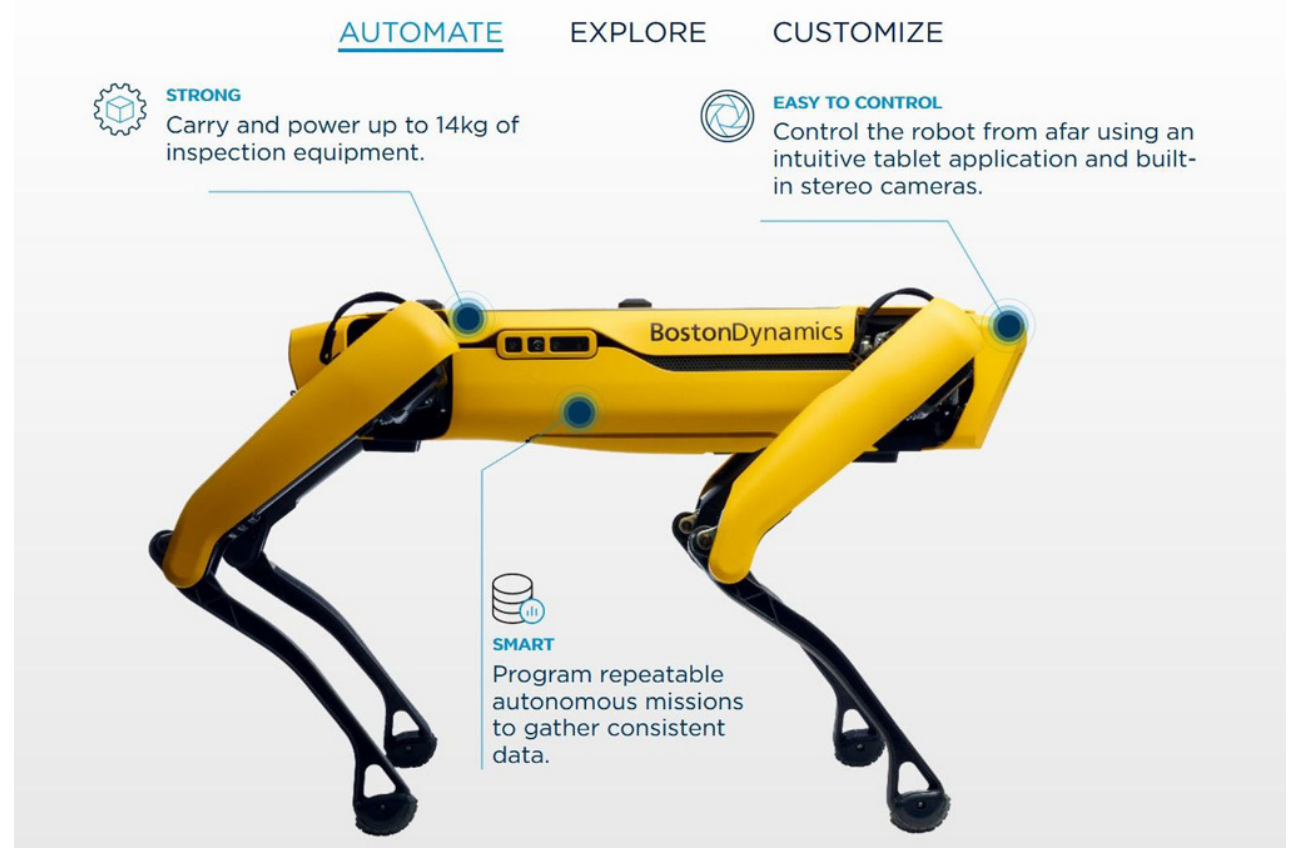

\subsection{Longwall Mining System}

Die modernsten Longwall Mining Systeme im Kohlenbergbau laufen in guten Bedingungen bis zu $98 \%$ im AutomatikBetrieb, überwacht aus der Leitzentrale [10]. Im Normalbetrieb ist so ein Kohlengewinnungsstreb also ein Roboter, der vom Menschen nur mehr überwacht wird.

Für die Installation und Wartung ist jedoch der Mensch vor Ort derzeit unersetzlich. Der Tausch von stumpfen Meisel oder defekten Kabeln oder Hydraulikelementen sind beispielsweise Aufgaben für das Wartungspersonal. Sollten größere Defekte oder geologische Störungen auftreten, muss zur Beseitigung Personal aus dem gesamten Betrieb zusammengezogen werden.
Daher ist das Longwall Mining System nur im störungsfreien Betrieb ein Roboter, der von seinem Wartungspersonal am Laufen gehalten wird. Der Roboter übernimmt aber die gesamte Gewinnungsarbeit und den Transport der Kohle aus dem Streb.

Auch hier zeigt sich der Bedarf für zukünftige mobile Wartungsroboter.

\subsection{Exploration}

Für die Exploration in gefluteten unterirdischen Hohlräumen wurde im europäischen Forschungsprojekt HORIZON 2020 UNEXMIN [11] der Explorationsroboter UX 1 (Abb. 1) 
Abb. 3: Der Sandvik Muldenkipper TH551itransportiert untertage mittels des AutoMine ${ }^{\circledR}$ Systems autonom ohne Fahrer seine Last durch das Bergbausystem (Quelle: Sandvik)

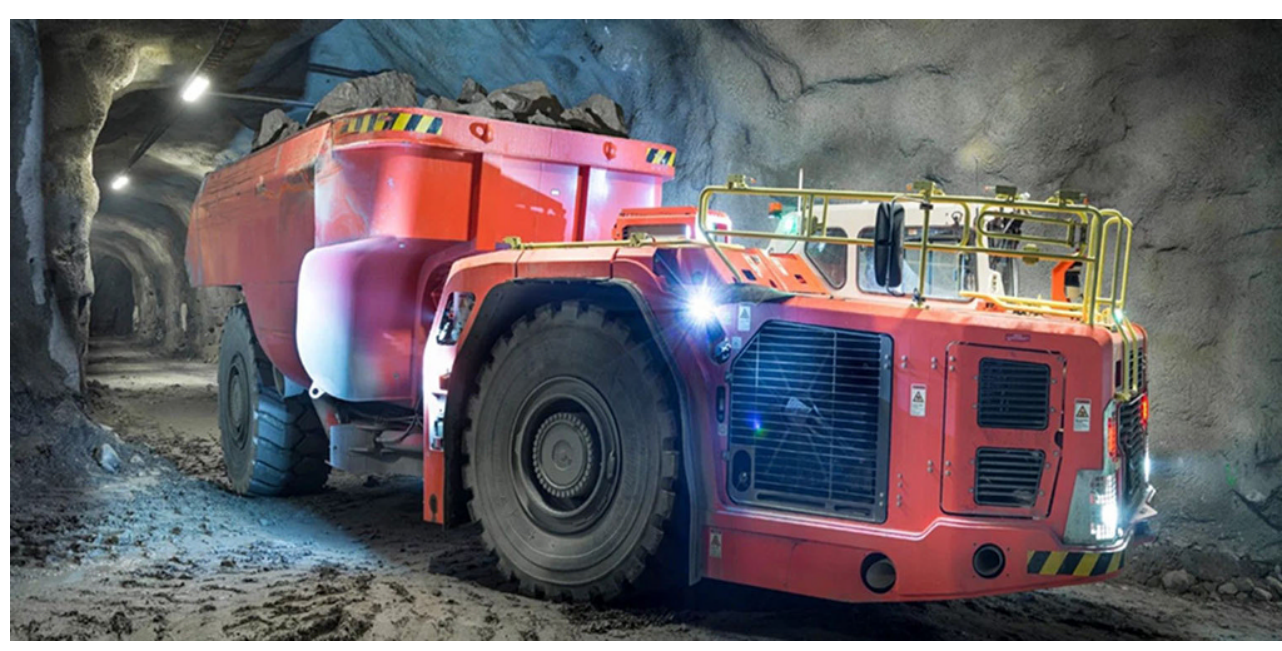

entwickelt. Dieser untersucht die gefluteten Bereiche optisch.

Das europäische Forschungsprojekt HORIZON 2020 ROBOMINERS [12] hat die Entwicklung von RoboterKonzepten für die Erkundung und Probenahme in tiefen Bergbauen, die auch geflutet sein können, zum Ziel. Ein ca. $1000 \mathrm{~g}$ schwerer Roboterprototyp wird entwickelt und im Labor erprobt. Die Montanuniversität Leoben ist für die Entwicklung der Materiallösekonzepte verantwortlich und auch für die Löseeinrichtung des Prototyps. Das Projekt ROBOMINERS wird noch an anderer Stelle dieser Ausgabe der BHM im Detail vorgestellt.

\subsection{Search and Rescue}

Nach Unglücken untertage ist ein direkter Zugang zum Ort des Geschehens oft für die Rettungskräfte zu gefährlich. Daher haben sich alle Rettungsorganisationen für den Bergbau schon früh für ferngesteuerte mobile Roboter interessiert, die der Gefahr durch giftige Gase oder Gesteinsfall ausgesetzt werden können [13].

In den Vereinigten Staaten von Amerika hat die Mine Safety and Health Administration (MSHA) in ihrer Mine Emergency Operations Division bei Bedarf auch kleine Suchroboter im Einsatz [14].

Auch China hat Search-and-Rescue Robotersysteme für den Einsatz im Kohlenbergbau entwickelt [15].

Als Beispiel für ein europäisches System sei hier der Mining-RoX der Technischen Universität Bergakademie Freiberg genannt [16].

Alle angeführten Beispiele haben als Fahrwerk entweder Räder oder Ketten. Das schränkt den Bewegungsradius auf gute Bodenverhältnisse ein. Besonders nach einem Steinfall ist der Fahrweg meist nicht mehr befahrbar.

\section{4 Überwachung gefährlicher Bereiche}

Daher eignen sich zur Befahrung schwieriger Bodenverhältnisse nach Steinfall Beine oder Schreitwerke besser.

Der schwedische Eisenerzkonzern LKAB gab im Oktober 2020 bekannt, dass er den Roboter SPOT ${ }^{\circledR}$ im Untertagebereich zur Erkundung von Schäden nach Gebirgsschlägen einsetzt [17]. SPOT ${ }^{\circledR}$ (Abb. 2) ist ein Roboter der Firma Boston Dynamics auf vier Beinen und wird um rund 75.000 US-Dollar angeboten. SPOT ${ }^{\circledR}$ kann bis zu 14 kg Messinstrumente aufnehmen und autonom in die Strecken vordringen.

\subsection{Transport - Autonome Fahrzeuge}

Das letzte Jahrzehnt sah die Einführung autonomer Muldenkipper, die ohne Menschen an Bord mit ihrer Nutzlast fahren - sowohl obertage wie auch untertage.

So berichtete der australische Eisenerzkonzern Fortescue Metals Group im September 2019, dass er seit der Einführung der "Autonomous haulage technology" von Caterpillar im Jahre 2012 in seinem Obertagebergbau in Pilbara bisher 137 autonome Muldenkipper zum Einsatz gebracht hatte. In dieser Zeit wurden mit dieser Flotte 33,5 Mio. Kilometer zurückgelegt und eine Milliarde Tonnen Material transportiert [18].

Caterpillar meldete im Mai 2020 weltweit 282 autonome Muldenkipper im Einsatz [19].

Auch Komatsu hat sein autonomes System erfolgreich auf den Markt zum Einsatz gebracht und vermeldete 2019 die Ausrüstung seines größten Muldenkippers 980E-4A mit $363 t$ Nutzlast für den autonomen Betrieb in einem Ölsandbergbau in Kanada [20].

Das AutoMine ${ }^{\circledR}$ System der Firma Sandvik (Abb. 3) ist für den autonomen und teleferngesteuerten Betrieb von Untertagefahrzeugen seit einem Jahrzehnt im Einsatz [21, 22].

Dabei ist es wichtig anzumerken, dass derzeit die Strecken, auf denen autonome Fahrzeuge unterwegs sind, aus 
Sicherheitsgründen für jeglichen Zutritt durch Menschen gesperrt werden, solange die Fahrzeuge unterwegs sind. Die Entwicklung entsprechender Systeme mit funktionaler Sicherheit, welche eine gleichzeitige Kooperation von Menschen und Roboter im selben Bereich zulassen, sind erst in den Anfängen.

\subsection{Autonome Bohrgeräte}

SowohI EPIROC als auch Sandvik bieten für Ober- und Untertage Produktionsbohrgeräte an, welche solange autonom bohren können, wie entsprechendes Material in den Karusselllafetten vorhanden ist [23]. Mit diesem autonomen Betrieb werden auch Zeiten des Schichtwechsels überbrückt, die Maschinen werden dabei meist mittels Teleoperation aus einer Überwachungszentrale beobachtet.

\section{Ausblick und Rolle der Montanuniversität Leoben}

Schrittweise halten also Arbeitsroboter Einzug in den Bergbau. Obwohl der Bedarf an Robotern für den Untertagebetrieb groß ist, hemmen die schwierigen Einsatzbedingungen und auch die begrenzten Forschungsmittel in der Bergbauindustrie raschere Fortschritte.

Eine Vorausschau in die Zukunft ist immer schwierig, aber der Autor erwartet, dass etwa gegen 2035 mobile Roboter für Installations- und Wartungsarbeiten für den Bergbau verfügbar sein werden. Damit kann dann in Teilbereichen untertage mit dem mannlosen Betrieb begonnen werden und Menschen müssen sich nur mehr in seltenen Ausnahmefällen in ungesunde und gefährliche Bereiche begeben.

Das HORIZON 2020 ROBOMINERS Projektkonsortium erwartet bis 2050 die Verfügbarkeit von Robotern für alle Bereiche im Bergbau.

Diese Transformation des Untertagebergbaus wird im Laufe des 21. Jahrhunderts stattfinden. Eine große sicherheitstechnische Herausforderung dabei ist die Kooperation von Menschen und Robotern zu Beginn im selben Arbeitsraum. Roboter müssen die Anwesenheit von Menschen erkennen können und entsprechend sicher operieren.

Die Aufgabe der Forschung und Lehre an der Montanuniversität Leoben ist es, einen Teil in der Anwendung von Robotern im Bergbau mit zu entwickeln und unsere Absolventen/innen auf die Anwendung von Robotern vorzubereiten. Als technische Universität und mit den laufenden Investitionen in die Digitalisierung, wie zum Beispiel den neuen Lehrstuhl für Cyber-Physical Systems und in anderen Bereichen, ist die Montanuniversität Leoben gut vorbereitet. Die Finanzierung für entsprechende Projekte einzuwerben, bleibt weiterhin eine Herausforderung, die es zu meistern gilt.

Nachhaltiger Bergbau beinhaltet auch, den Menschen in gefährlichen Bereichen durch Roboter zu ersetzen. Das ist keine einfache Aufgabe, aber wir müssen sie Schritt für Schritt lösen.
Funding. Open access funding provided by Montanuniversität Leoben.

Open Access Dieser Artikel wird unter der Creative Commons Namensnennung 4.0 International Lizenz veröffentlicht, welche die Nutzung, Vervielfältigung, Bearbeitung, Verbreitung und Wiedergabe in jeglichem Medium und Format erlaubt, sofern Sie den/die ursprünglichen Autor(en) und die Quelle ordnungsgemäß nennen, einen Link zur Creative Commons Lizenz beifügen und angeben, ob Änderungen vorgenommen wurden.

Die in diesem Artikel enthaltenen Bilder und sonstiges Drittmaterial unterliegen ebenfalls der genannten Creative Commons Lizenz, sofern sich aus der Abbildungslegende nichts anderes ergibt. Sofern das betreffende Material nicht unter der genannten Creative Commons Lizenz steht und die betreffende Handlung nicht nach gesetzlichen Vorschriften erlaubt ist, ist für die oben aufgeführten Weiterverwendungen des Materials die Einwilligung des jeweiligen Rechteinhabers einzuholen.

Weitere Details zur Lizenz entnehmen Sie bitte der Lizenzinformation auf http://creativecommons.org/licenses/by/4.0/deed.de.

\section{Literatur}

1. Berner M.; Sifferlinger N.A.; Moser P.: Reduction of personnel in exposed areas, safety devices and early steps towards robots in mining, in: Conference Proceedings of International Future Mining Conference 2019, Sydney, The Australian Institute for Mining and Metallurgy Publication Series No 772019, Australia, 2019, S. 29-33

2. Marshall, J. A.; Bonchis, A.; Nebot, E.; Scheding, S.: Robotics in Mining, in: Springer Handbook of Robotics, $2^{\text {nd }}$ Edition, Berlin, Heidelberg: Springer Verlag, 2016, S.1549-1576

3. Sandvik: AutoMine®, https://www.rocktechnology.sandvik/en/prod ucts/automation/automine-equipment-and-teleoperation-systems/ automine-underground/. Zugegriffen: 17.11.2020

4. Robotic Industries Association: Humanoid Robots; https://www. robotics.org/service-robots/humanoid-robots. Zugegriffen: 20.11.2020

5. DARPA: Creating breakthrough technologies and capabilities for national security; https://www.darpa.mil/. Zugegriffen: 23.11.2020

6. Spenko, M.; Buerger, S.; lagnemma, K. (Eds): The DARPA Robotics Challenge Finals: Humanoid Robots To The Rescue, Cham: Springer, 2018

7. DARPA: Subterranean Challenge Identifies Qualified Teams for Cave Circuit Virtual Competition; 29 September 2020; https://www.darpa. mil/news-events/2020-09-28a. Zugegriffen: 23.11.2020

8. Military.com: Army is spending half a billion to train soldiers to fight underground; https://www.military.com/daily-news/2018/ 06/24/army-spending-half-billion-train-troops-fight-underground. html. Zugegriffen: 23.11.2020

9. Boston Dynamics: Changing your ideas what robots can do; https:// www.bostondynamics.com/. Zugegriffen: 23.11.2020

10. CAT Longwall Automation, https://www.cat.com/en_US/byindustry/mining/articles/longwall-automation-download.html. Zugegriffen: 18.11.2020

11. HORIZON 2020 UNEXMIN: An autonomous underwater explorer for flooded mines; https://www.unexmin.eu/. Zugegriffen: 23.11.2020

12. HORIZION 2020 ROBOMINERS: Resilient bio-inspired modular robotic miners; https://robominers.eu/. Zugegriffen: 24.11.2020

13. Hemanth Reddy, A.; Balla Kalyan; Murthy, C.S.N: Mine rescue robot system - a review, Procedia Earth and Planetary Science, 11 (2015), S. $457-462$

14. MSHA: Mine Emergency Operations Divisions; https://www.msha. gov/about/program-areas/technical-support/pittsburgh-safety-heal th-technology-center. Zugegriffen: 29.11.2020

15. Jingchao Zhao; Junyao Gao; Fangzhou Zhao; Yi Liu: A Search-andRescue Robot System for Remotely Sensing the Underground Coal Mine Environment; in: Sensors (Basel) (2017) 2017 October 17 (10), S. $2626-2452$ 
16. TU Freiberg: Robots in Mining; https://tu-freiberg.de/fakult1/inf/ professuren/virtuelle-realitaet-und-multimedia/forschung/projekte/ robots-in-mining. Zugegriffen: 25.11.2020

17. LKAB: Unique robotic dog serving faithfully at LKAB, News 21 October 2020; https://www.lkab.com/en/news-room/news/uniquerobotic-dog-serving-faithfully-at-lkab/. Zugegriffen: 23.11.2020

18. Mining Magazine: FMG hauls more than one billion tonnes autonomously, 13 September 2019; https://www.miningmagazine. com/fleet/news/1371548/fmg-hauls-more-than-one-billion-tonnesautonomously. Zugegriffen: 18.11.2020

19. CAT: A world leader in autonomous mining, 26 May 2020, https:// www.caterpillar.com/en/news/caterpillarNews/customer-dealerproduct/a-world-leader-in-autonomous-mining.html. Zugegriffen: 19.11.2020

20. Australian Mining: Komatsu unveils largest autonomous truck, 24 April 2019; https://www.australianmining.com.au/news/komatsusunveils-largest-autonomous-truck/. Zugegriffen: 18.11.2020
21. Sandvik: Automine Equipment Automation and Teleoperation Systems; https://www.rocktechnology.sandvik/en/products/automation/ automine-equipment-and-teleoperation-systems/. Zugegriffen: 25.11.2020

22. Sandvik: Sandvik continues Codelco digitalization partnership delivering additional automation at El Teniente mine; https://www. rocktechnology.sandvik/en/news-and-media/news-archive/2019/05/ sandvik-continues-codelco-digitalization-partnership-deliveringadditional-automation-at-el-teniente-mine/. Zugegriffen: 25.11.2020

23. EPIROC: Machine autonomy; https://www.epiroc.com/en-bf/applica tions/mining/automation/machine-autonomy. Zugegriffen: 25.11.2020

Hinweis des Verlags. Der Verlag bleibt in Hinblick auf geografische Zuordnungen und Gebietsbezeichnungen in veröffentlichten Karten und Institutsadressen neutral. 